\title{
Immunophenotyping pattern of mixed phenotypic acute leukemia : historical review and diagnostic pitfalls
}

\author{
Monika Gupta $^{1}$, Pooja Dhamija ${ }^{1}$, Nisha Marwah ${ }^{1}$, Sujata Kumari SP Prasad ${ }^{1}$, \\ Rajeev Sen ${ }^{1}$ \\ ${ }^{I}$ Department of Pathology, Pandit Bhagwat Dayal Sharma University of Health Sciences, India
}

\section{Keywords:}

Flowcytometry; Immunophenotyping; Mixed Phenotypic; Acute Leukemia;

\begin{abstract}
Background: Multiparametric approaches are used for the diagnosis and classification of acute leukemia. Even after extensive immunophenotyping, a rare and heterogeneous subgroup that cannot be readily classified as Mixed Phenotypic Acute leukemia in which the blasts exhibit the antigens of more than one lineage. This study aimed to analyze the incidence of Mixed Phenotypic Acute Leukemia and correlate the clinicopathological and immunophenotypic characteristics.
\end{abstract}

Materials and Methods: This study was done in a tertiary health institute. Complete clinical details of patients were taken followed by morphological evaluation including peripheral blood, bone marrow aspiration, and biopsy was done. Immunophenotyping was performed on the FACS Canto II Flow cytometer. The diagnosis of Mixed Phenotypic Acute Leukemiawas based on the World Health Organization 2008 classification.

Results: We analyzed 7 cases, which include 57.14\%of B/Mixed Phenotypic Acute Leukemia and 42.86\% of T/Mixed Phenotypic Acute Leukemia. The male to female ratio was 0.4:1. Hematological parameters including hemoglobin, total leucocyte count, platelets count, and blasts percentage were correlated. On flow cytometry, all cases were positive for cMPO while B/Myeloid cases were positive for CD19 and at least one $\mathrm{B}$ cell marker. T/Myeloid cases were positive for cCD3 and CD7 in all cases besides other myeloid and $\mathrm{T}$ cell markers.

Conclusions: The frequency of Mixed Phenotypic Acute Leukemia in our study was found to be $2.7 \%$, in concordance with published data (2.2-2.6\%). Strict diagnostic criteria should be followed in the diagnosis because it originates from primitive multipotent progenitors which are resistant to treatment and lineage plasticity.

\section{Correspondence:}

Dr. Monika Gupta, MD

Associate Professor, Department of Pathology,

Pandit Bhagwat Dayal Sharma University of Health Sciences, India ORCID ID: 0000-0003-3438-2943

Email: monikashashwat@hotmail.com

Reveived : January $2^{\text {nd }} 2021 ;$ Accepted : June $4^{\text {th }} 2021$

Citation : Gupta M, Dhamija P, Marwah N, Prasad SKSP, Sen R. Immunophenotyping Pattern of Mixed Phenotypic Acute Leukemia.Journal of Pathology of Nepal.202;11(2):1824-9. DOI: 10.3126/ jpn.v11i2.34001

Copyright: This is an open-access article distributed under the terms of the Creative Commons Attribution 4.0 International License, which permits unrestricted use, distribution, and reproduction in any medium, provided the original author and source are credited.

\section{INTRODUCTION}

Acute leukemia (AL) is a clonal hematopoietic stem cells disorder characterized by an increase in immature cells $(>20 \%)$ in the bone marrow (BM) and/or peripheral blood (PB). Multiparametric approaches are being used for the diagnosis and sub-classification of AL like morphological assessment, immunophenotyping, karyotyping, and molecular genetics analyses. The first step in the classification of $\mathrm{AL}$ is to assign lineage by the resemblance to normal progenitor cells. Most cases are unequivocally assigned either myeloid or lymphoid lineage based on the expression of a set of antigens. ${ }^{1,2}$ 
However, even after extensive immunophenotyping, a rare and heterogeneous subgroup of AL cannot be readily classified. Leukemias falling into this category have been given many different names, including acute mixed lineage leukemia, bi-phenotypic leukemias, hybrid leukemias, undifferentiated leukemias, and, most recently, leukemia of ambiguous lineage. ${ }^{3}$ One of this subgroup of ALis Mixed Phenotypic Acute leukemia(MPAL)in which the blasts exhibit the antigens of more than one lineage. MPAL can be B/Myeloid, T/Myeloid, or B/T. The mixed phenotype in $\mathrm{T} /$ myeloid or B/myeloid MPAL can occur in three ways:

1.Two distinct blast populations, one showing immunophenotype of acute myeloid leukemia (AML) and the other showing immunophenotype of lymphoid blast population,

2. Single population of blasts meeting criteria of B-acute lymphoblastic leukemia (ALL)/T-ALL with an expression of myeloperoxidase (MPO),

3. Single population of blasts meeting criteria of B-ALL/TALL with evidence of monocytic differentiation. ${ }^{4}$

It is a rare disease and comprises $2-5 \%$ of AL. ${ }^{5}$ This retrospective study aimed to analyze the incidence of MPAL and correlate the clinicopathological and immunophenotypical characteristics.

\section{MATERIALS AND METHODS}

This study was conducted in PT. B.D. Sharma Institute of Health Sciences, Rohtak from2016 to June 2020. Clinical details, morphological evaluation, immunophenotyping data were retrieved. The diagnosis of MPAL was based mainly on the WHO 2008 classification. ${ }^{4}$ A serial number was assigned to each one to ensure confidentiality. Ethical approval for this study was obtained from the institutional ethics committee.

During this period 256 cases of AL were analyzed. In all patients, air-dried peripheral blood smears and bone marrow aspirates were stained with Giemsa stain. To study the blast morphology, cytochemical stains like MPO, Sudan, periodic acid Schiff (PAS), nonspecific esterase were performed. Cases were classified as ALL or AML by morphology and cytochemistry.

Flowcytometric analysis (FCA) was done using the monoclonal antibody panel of AL on peripheral blood/ bone marrow using a standard stain-lyse-wash method. The fluorescent probes used were Allophycocyanin (APC-H7) for CD45, Flouroisothiocyanate (FITC), Phycoerythrin (PE), Allophycocyanin (APC), Peridinin-chlorophyll protein (PerCP), and PerCPCy5.5. FCA was performed using primary and/or a secondary panel of monoclonal antibodies. The $\mathrm{CD}$ panel comprised of CD45, CD34, sCD3, cyCD3, CD5, CD2, CD7, CD4, CD8, CD10, CD20, CD19, CD79a, Tdt, CD13, CD33, CD117, HLA-DR, MPO, CD64.

Listmode data were acquired on FACS Canto II Flowcytometer (Becton Dickinson, San Jose, CA) and analyzed by FACS Diva software. Blasts were characterized by low side scatter, diminished or low CD45 expression, and CD34 positivity. In case CD34 is negative in the blast window, gating was done by using other immaturity markers as CD117 and CD 19/10 co-expression. For interpretation, we used a cut-off of $20 \%$ for surface markers and $10 \%$ for cytoplasmic markers.

\section{RESULTS}

In our study, we came across 7 MPAL cases, which include 4 cases $(57.14 \%)$ of B/MPALand 3 cases $(42.86 \%)$ of $\mathrm{T} /$ MPAL. There were 2 males and 5 females with an M: F ratio of $0.4: 1$. The mean age was 39 years (range 23-70 years). The common clinicopathological findings were anemia $(100 \%)$, fever $(71.4 \%)$, bony tenderness $(42.8 \%)$, and organomegaly $(85.7 \%)$ cases(Table 1$)$.

Hematologicalparameters included mean hemoglobin $(\mathrm{Hb})$ $6.3 \mathrm{~g} \%(4.3-8.0 \mathrm{~g} \%)$, mean total leucocyte count (TLC) $137.8 \times 109 / \mathrm{L}(27 \times 109 / \mathrm{L}-330 \times 109 / \mathrm{L})$, mean platelets $56.8 \times 109 / \mathrm{L}(20 \times 109 / \mathrm{L}-80 \times 109 / \mathrm{L})$, mean percentage of blasts $77 \%(66 \%-90 \%)$ in peripheral smear(Table 2).

Morphological evaluation of $\mathrm{PB} / \mathrm{BM}$ did not reveal any characteristic morphological appearance of the blasts. Six

Table 1: Clinical Findings in Mixed Phenotypic Acute leukemia

\begin{tabular}{|c|c|c|c|c|c|c|c|c|}
\hline Sex & Age (years) & Fever & Bleeding sites & Bony tenderness & Pallor & $\mathrm{LN}$ & Liver & Spleen \\
\hline $\mathrm{F}$ & 23 & + & - & + & + & - & - & - \\
\hline $\mathrm{F}$ & 40 & - & + & + & + & + & - & + \\
\hline $\mathrm{M}$ & 40 & + & - & - & + & + & + & + \\
\hline $\mathrm{F}$ & 13 & + & + & - & + & + & + & + \\
\hline M & 30 & + & - & + & + & - & - & + \\
\hline $\mathrm{F}$ & 70 & + & - & - & + & - & + & + \\
\hline $\mathrm{F}$ & 60 & - & - & - & + & - & - & + \\
\hline
\end{tabular}


Table 2: Laboratory Findings in Mixed Phenotyic Acute leukemia

\begin{tabular}{cccccccc}
\hline $\mathrm{Hb}(\mathrm{g} \%)$ & $\mathrm{TLC} \times 109 / \mathrm{L}$ & Platelets $\times 109 / \mathrm{L}$ & PB blasts $(\%)$ & Morphology $(\mathrm{BMA})$ & MPO & PAS & SBB \\
\hline 4.3 & 330 & 40 & $70 \%$ & AML & + & + & + \\
\hline 7.8 & 200 & 40 & $80 \%$ & AML & + & + \\
\hline 8.0 & 100 & 80 & $70 \%$ & ALL & - & + \\
\hline 6.6 & 190 & 58 & $77 \%$ & AML & + & + & + \\
\hline 5.5 & 98 & 80 & $90 \%$ & AML & + & + \\
4.3 & 27 & 80 & $66 \%$ & AML & + & + & + \\
7.8 & 197 & 20 & $87 \%$ & AML & + & + & + \\
\end{tabular}

PB: peripheral blood; BMA: bone marrow aspiration, SBB: Sudan Black B
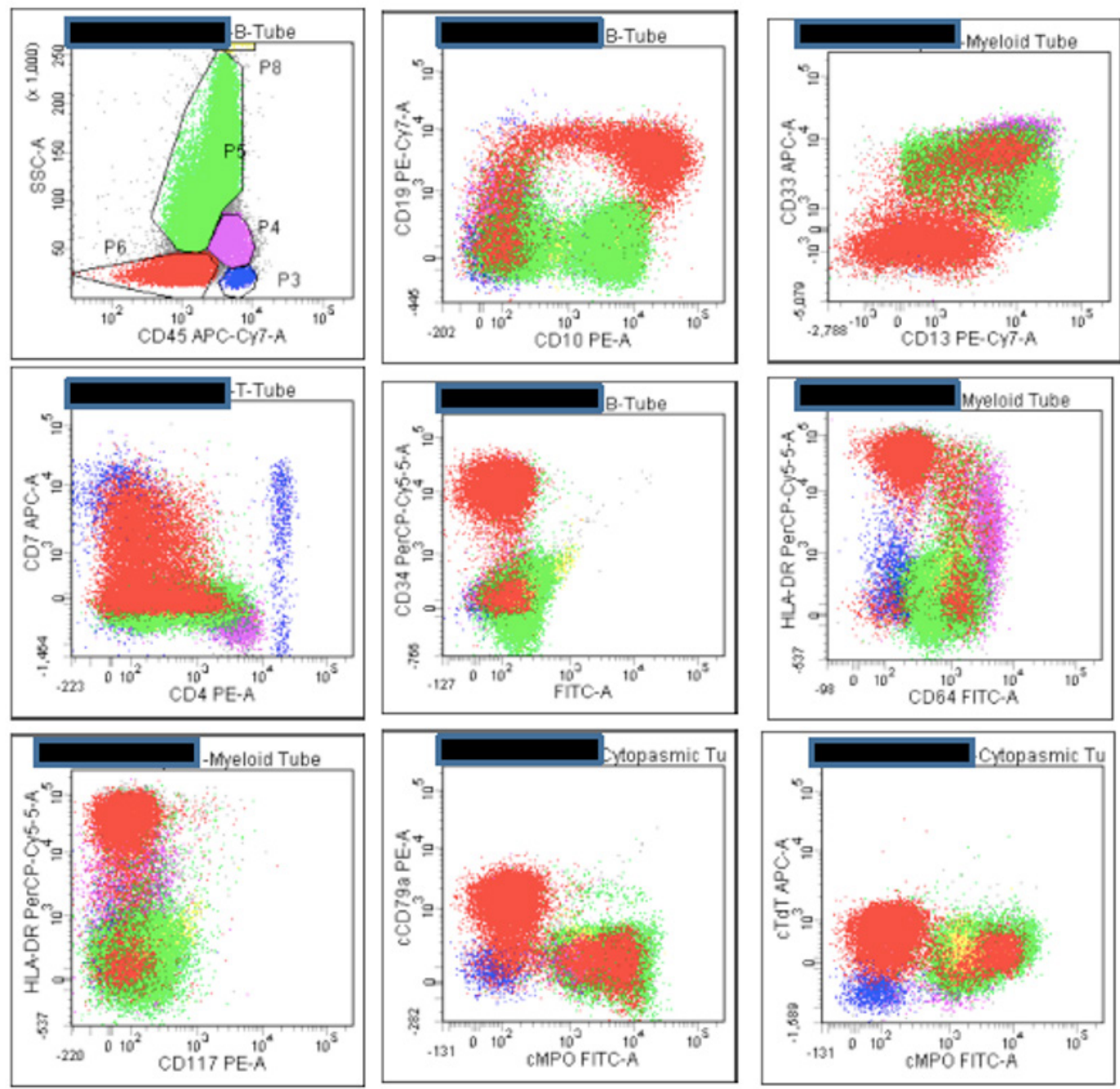

Figure 1: MPAL B/Myeloid: Blasts are positive for CD10, CD19, CD34,CD13, CD33, HLA-DR, CD7, cCD79a, cMPO, Tdt and Dim positive for CD45, negative for CD117 and CD64

cases were diagnosed as AML due to the positivity of MPO and Sudan on cytochemistry and one case was negative for both stains diagnosed as ALL (fig 1).

On flow cytometry, all cases were positive for cMPO while $\mathrm{B} /$ Myeloid cases were positive for CD19 and at least on B cell marker. T/Myeloid cases were positive for $\mathrm{cCD} 3$ as well as CD7 in all cases besides other myeloid and T cell markers (Table 3 ).

In our study, all 7 patients started with chemotherapy. As all of them were adult patients Hyper- fractionated cyclophosphamide, vincristine, doxorubicin, and dexamethasone(Hyper CVAD) regimen was given. Only two patients achieved complete remission after one month while one patient achieved late complete remission. Four 
Table 3: Immunophenotyping details in Mixed Phenotypic Acute leukemia

\begin{tabular}{|c|c|c|c|c|c|}
\hline \multicolumn{2}{|c|}{ Myeloid markers } & \multicolumn{2}{|c|}{ Lymphoid markers } & \multirow{2}{*}{ Immaturity markers } & \multirow{2}{*}{ Diagnosis } \\
\hline Specific & Others & Specific & Others & & \\
\hline cMPO & $\begin{array}{l}\text { CD13,CD33,CD64, } \\
\text { CD117 }\end{array}$ & CD19 & CD79a, CD4 & CD34, HLA-DR & B/Myeloid \\
\hline cMPO & $\mathrm{CD} 13, \mathrm{CD} 33, \mathrm{CD} 64$ & CD19 & $\mathrm{CD} 79 \mathrm{a}, \mathrm{CD} 10, \mathrm{CD} 20$ & CD34, HLA-DR, Tdt & B/Myeloid \\
\hline cMPO & $\mathrm{CD} 13, \mathrm{CD} 33$ & CD19 & CD79a, CD10 & CD34, HLA-DR, Tdt & B/Myeloid \\
\hline cMPO & $\mathrm{CD} 13, \mathrm{CD} 33$ & CD19 & $\mathrm{CD} 79 \mathrm{a}, \mathrm{CD} 10, \mathrm{CD} 20$ & CD34, HLA-DR, Tdt & B/Myeloid \\
\hline cMPO & CD13, CD33, CD117 & $\mathrm{cCD} 3$ & CD7 & CD34, HLA-DR, Tdt & T/Myeloid \\
\hline cMPO & $\begin{array}{l}\text { CD13,CD33,CD64, } \\
\text { CD117 }\end{array}$ & $\mathrm{cCD} 3$ & $\mathrm{CD} 7, \mathrm{CD} 4, \mathrm{CD} 5$ & CD34, HLA-DR, Tdt & T/Myeloid \\
\hline cMPO & $\mathrm{CD} 13, \mathrm{CD} 33, \mathrm{CD} 117$ & $\mathrm{cCD} 3$ & CD7,CD5 & CD34, HLA-DR & T/Myeloid \\
\hline
\end{tabular}

patients were given palliative chemotherapy because of age and financial constraints. All these four patients expired during treatment.

\section{DISCUSSION}

The first published reports on biphenotypicAL occurred in the 1980s when monoclonal antibodies were first being used to characterize leukemic cells. A defined scoring system for biphenotypicAL was proposed by Catovsky et al. ${ }^{6}$ The European Group for the immunological characterization of leukemias (EGIL) later proposed an immunological classification and characterization of AL, which included a definition for biphenotypicAL. BiphenotypicAL was defined by EGIL when a score over 2 points was achieved for the myeloid as well as one of the lymphoid lineages. At least $20 \%$ of cells staining with a monoclonal antibody was chosen as a cut-off point to consider a marker as positive. ${ }^{7}$ An exception was made for CD3, MPO, CD 79a, and Tdt because of their high degree of specificity, this is not adopted by World Health Organization (WHO). ${ }^{1}$ (Table 4)WHO classification proposed a simpler diagnostic algorithm based on more specific lineage markers. New consensus criteria for MPAL were published in the 4th edition of the WHO classification of tumors of hematopoietic and lymphoid tissues and remain essentially unchanged in the 2016 update

\begin{tabular}{|c|c|c|c|}
\hline Points & B & $\mathbf{T}$ & Myeloid \\
\hline \multirow{3}{*}{2} & CyCD79a & CD3(sm/cy) & MPO \\
\hline & CyCD22 & TCR-œß & \\
\hline & CyIgM & $\mathrm{TCR}-\gamma \delta$ & CD79a,CD10,CD20 \\
\hline \multirow{4}{*}{1} & CD19 & $\mathrm{CD} 2$ & CD117 \\
\hline & CD20 & CD5 & CD13 \\
\hline & CD10 & $\mathrm{CD} 8$ & CD33 \\
\hline & & $\mathrm{CD} 10$ & CDw65 \\
\hline \multirow{3}{*}{0.5} & $\mathrm{TdT}$ & Tdt & CD14 \\
\hline & $\mathrm{CD} 24$ & $\mathrm{CD} 7$ & CD15 \\
\hline & & CD1a & CD64 \\
\hline
\end{tabular}

to the classification WHO criteria. They emphasize a few key lineage-defining markers with particular emphasis on CD19 for B lineage, CD3 for T lineage, and MPO for myeloid lineage4 (Table 5).

For all practical purposes, the WHO approach in the diagnosis of MPAL mainly relies on a few markers on flow cytometric immunophenotyping. Other additional methodology includes immunohistochemistry and cytochemistry which can at times be helpful. ${ }^{8}$

MPAL is thought to arise from a multipotential hemopoietic stem cell that has the potential to differentiate into any lineage. Most of the reported cases of MPAL express early hematopoietic markers CD34 and HLA-DR suggesting an early precursor stem cell origin. Another explanation for the development of MPAL is that the blasts originate from a lymphoid precursor that has reactivated a myeloid differentiation program. ${ }^{5,10}$ BCR-ABL1 fusion and KMT2A translocation are two genetically defined categories in MPAL. KMT2A translocations are more common in pediatric MPALwhile BCR-ABL1 is more common in adults. ${ }^{2,9}$

The WHO classification of MPAL specifically excludes the entities which are covered in the proposed AML

\begin{tabular}{|c|c|}
\hline Lineage & Markers \\
\hline Myeloid & $\begin{array}{l}\text { MPO (Flow cytometry, immunohistochemistry, or } \\
\text { enzyme cytochemistry) } \\
\text {-OR- } \\
\text { Monocytic differentiation (at least } 2 \text { of the } \\
\text { following: NSE cytochemistry, CD11c, CD14, } \\
\text { CD64, lysozyme) }\end{array}$ \\
\hline $\mathrm{T}$ lineage & $\begin{array}{l}\text { Strong cytoplasmic CD3 } \\
\text {-OR- } \\
\text { Surface CD3 }\end{array}$ \\
\hline B lineage & $\begin{array}{l}\text { Strong CD19 with at least } 1 \text { of the following strongly } \\
\text { expressed: CD } 79 \text { a, cytoplasmic CD22, or CD } 10 \\
\text {-OR- } \\
\text { Weak CD19 with at least } 2 \text { of the following strongly } \\
\text { expressed: CD } 79 \text { a, cytoplasmic CD22, or CD10 }\end{array}$ \\
\hline
\end{tabular}


classification of leukemias such as $\mathrm{t}(8 ; 21), \mathrm{t}(15 ; 17), \operatorname{inv}(16)$, FGFR1 mutations/ translocations, blast phase of chronic myelogenous leukemia, AML with myelodysplasia-related changes (AML-MRC), and therapy-related AML with complex karyotype. ${ }^{2}$

Even after WHO guidelines for the diagnosis of MPAL, there are certain pitfalls. i) AML MRC should be excluded by careful patient history and correlation with the blood and bone marrow morphology. ii) WHO classification does not specify thresholds for the interpretation of MPO expression. Its expression and varying techniques used for detection are left on the individual laboratories to decide. However, it is very critical for distinguishing between ALL and MPAL. iii) Overlooking minor populations of monocytic blasts, lymphoid blasts, or myeloid blasts that are diagnostic of bilinear AL because it is high risk with the sudden proliferation of a minor clone during induction therapy. ${ }^{2,9}$

Most of our cases were diagnosed on flow cytometry. The frequency of MPAL in our study was found to be $2.7 \%$ (7/256). This is in concordance with published data, which documents the frequency ranging from 2.2 to $2.6 \% .{ }^{11-15}$ There was a slight female preponderance with $\mathrm{M}$ : F ratio of $0.4: 1$ which is in contrast to the studies done by Charles et $\mathrm{al}^{2}$ and Pawar et $\mathrm{al}^{8}$ which showed a male predominance.

During the study period, a diagnosis of MPAL was made in 7 patients. B/ myeloid was the predominant type with four $(4 / 7)$ cases, which is consistent with the majority of the published studies where $\mathrm{B} /$ myeloid constitutes the predominating type of MPAL ${ }^{10,14}$ while Sukumaran et al 5reported T/myeloid as the predominant type of MPAL in their study.

MPAL is derived from the early stages of hematopoietic differentiation which are capable of differentiating into myeloid and lymphoid lineages. CD34 and HLA-DR is a marker of early hematopoietic cells and their expression was seen in $100 \%$ of case in our study. These findings were in concordance with the studies done by Charles et $\mathrm{al}^{2}$ and Pawar et $a .^{8}$ Pawar et $\mathrm{al}^{8}$ reported higher mean $\mathrm{Hb}$ and platelet count while TLC and peripheral blast count were lower than our study. The difference may be due to the fewer number of patients in the present observation.

The most important concern for the diagnostic pathologist approaching a new case of AL is to provide necessary and sufficient information for initial treatment at the earliest. Outcomes for MPAL were generally worse than for comparison cohorts of patients with AML and ALL due to high-risk genetics. Most reports show a better initial response to ALL-directed chemotherapy than AML directed therapies. ${ }^{2,16,17}$ This makes sense because most AL that fit the current WHO classification of MPAL more closely resembles ALL because of genetics and immunophenotype.
There were a few limitations of this study like a small number of patients, lack of cytogenetics and molecular studies (PCR) in these cases to draw an important conclusion. However, a comprehensive panel of antibodies is a must for the correct diagnosis of MPAL.

\section{CONCLUSIONS}

MPALisa rare leukemia with diagnostic and therapeutic challenge and worse prognosis due to origin from primitive multipotent progenitors which are resistant to treatment and lineage plasticity. Strictdiagnostic criteria should be followed in the diagnosis. Immunophenotyping is essential and should include lineage-specific markers along with the cytoplasmic markers for proper categorization of MPAL. Even though cytogenetics and molecular analysis for further prognostic stratification and treatment, are available, treating such patients remain a challenge even today.

\section{Conflict of interest: None}

\section{REFERENCES}

1. Weinberg OK, Arber DA. Mixed-phenotype acute leukemia: historical overview and a new definition. Leukemia. 2010;24:184451. Crossref

2. Charles NJ, Boyer DF. Mixed-Phenotype Acute Leukemia Diagnostic Criteria and Pitfalls. Arch Pathol Lab Med. 2017;141:1462-68. Crossref

3. Weir EG, Borowitz MJ. Acute leukemias of ambiguous lineage. In: Jaffe ES, Harris NL, Vardiman JW, Campo E, Arber DA (eds). Hematopathology. Elsevier: Philadelphia, 2010. $\underline{\text { Crossref }}$

4. Borowitz MJ, Bene MC, Harris NL, et al. Acute leukemias of ambiguous lineage. In: Swerdlow SH, Campo E, Harris NL, Jaffe ES, Pileri SA, Stein H, Thiele J, Vardiman JW (eds). WHO Classification of Tumours of Haematopoietic and Lymphoid Tissues. IARC Press: Lyon, 2008, pp 150-155.

5. Sukumaran R, Nair RA, Jacob PM, et al. Flow cytometric analysis of Mixed phenotype acute leukemia: Experience from a tertiary oncology center. Indian J Pathol Microbiol 2015;58:18 Crossref

6. Catovsky D, Matutes E, Buccheri V, et al. A classification of acute leukemia for the 1990s. Ann Hematol 1991;62:16-21. Crossref

7. Bene MC, Castoldi G, Knapp W, et al. Proposals for the immunological classification of acute leukemias. European Group for the Immunological Characterization of Leukemias (EGIL). Leukemia 1995; 9: 1783-86. Website

8. Pawar RN, Banerjee S, Bramha S, et al. Mixed-phenotypic acute leukemia series from tertiary care center. Indian J PatholMicrobiol2017;60:43-9. Website

9. Khurshid A, Khan SA, Altaf C, et al. Immunophenotyping Pattern in Mixed Phenotype Acute Leukaemias. Pak Armed Forces Med J 2017;67:883-9. Website

10. Gujral S, Polampalli S, Badrinath Y, et al. Clinicohematological profile in biphenotypic acute leukemia.Indian J Cancer 2009;46:160$8 \underline{\text { Crossref }}$

11. Matutes E, Pickl WF, Van't Veer M,et al. Mixed phenotype acute leukemia: Clinical and laboratory features and outcome in 100 patients defined according to the WHO 2008 classification. Blood 2011;117:3163 71. Crossref 
12. Yan L, Ping N, Zhu M,et al. Clinical, immunophenotypic, cytogenetic, and molecular genetic features in 117 adult patients with mixed phenotype acute leukemia defined by WHO 2008 classification. Haematologica2012;97:1708 12. Crossref

13. Owaidah TM, Al Beihany A, Iqbal MA, et al. Cytogenetics, molecular and ultrastructural characteristics of biphenotypic acute leukemia identified by the EGIL scoring system. Leukemia2006;20:620 6 . Crossref

14. Xu XQ, Wang JM, Lü SQ, et al. Clinical and biological characteristics of adult biphenotypic acute leukemia in comparison with that of acute myeloid leukemia and acute lymphoblastic leukemia: A case series of a Chinese population. Haematologica 2009;94:919 27. Crossref
15. Legrand O, Perrot JY, Simonin G, et al. Adult biphenotypic acute leukaemia: An entity with poor prognosis which is related to unfavourable cytogenetics and $\mathrm{P}$ glycoprotein over expression. Br J Haematol1998;100:147 55. $\underline{\text { Crossref }}$

16. Gerr H, Zimmermann M, Schrappe M, et al. Acute leukaemias of ambiguous lineage in children: characterization, prognosis and therapy recommendations. Br J Haematol. 2010;149:84-92. Crossref

17. Rubnitz JE, Onciu M, Pounds S, et al. Acute mixed lineage leukemia in children: the experience of St Jude Children's Research Hospital. Blood. 2009;113:5083-9. Crossref 